\title{
Optogenetic manipulation of dorsal raphe serotonergic neurons modulates emotional behaviors in rodents
}

\author{
Naoya Nishitani ${ }^{1}$, Nozomi Asaoka ${ }^{1}$, Hiroyuki Kawai ${ }^{1}$, Norihiro Shibui ${ }^{1}$, Yuma Nagai ${ }^{1}$, Chihiro Andoh ${ }^{1}$, \\ Kazuki Nagayasu', Hisashi Shirakawa', Takayuki Nakagawa ${ }^{2}$, Shuji Kaneko ${ }^{1}$ \\ ${ }^{I}$ Department of Molecular Pharmacology, Graduate School of Pharmaceutical Sciences, Kyoto University, Japan, \\ ${ }^{2}$ Department of Clinical Pharmacology and Therapeutics, Kyoto University Hospital, Japan
}

Serotonergic neurons are thought to play an important role in the control of the emotional states. Optogenetics using channelrhodopsin-2 (ChR2), a light activated cation channel, and archaerhodopsin (eArchT), a light-activated proton pump, have been widely used for the accurate control of the neuronal activity by light. Specifically, importance of the activity of serotonergic neurons in the control of anxiety has been shown by optogenetic analyses in transgenic mice. However, elucidation of the behavioral differences among mice strains and the animal species necessitates the tools for selective control of serotonergic neurons across the strains and the species. Here, we made a lentiviral vector (LVV) capable of optogenetic control of serotonergic neurons in mice and rats. In the dorsal raphe nucleus (DRN), one of the serotonergic nucleus, of mouse and rat injected with the LVV, transgenes were selectively expressed in tryptophan hydroxylase (TPH)-positive, serotonergic neurons. In whole-cell recordings from ChR2-positive and eArchT-positive neurons in acute DRN slice, photostimulation evoked action potentials and inhibited neuronal firing, respectively. Optogenetic stimulation of the mouse DRN serotonergic neurons decreased immobility duration in the tail suspension test, whereas it had no effect on the locomotor activity and anxiety-like behaviors in open field test and elevated plus maze test. Moreover, Optogenetic stimulation of the rat DRN serotonergic neurons decreased immobility duration in the forced swim test, while optogenetic inhibition of them enhanced anxiety-like behaviors in elevated plus maze test. These results demonstrate that selective activation of DRN serotonergic neurons is sufficient to elicit antidepressant-like effect in mice and rats, and that these new vectors pave the way towards selective manipulation of serotonergic neural circuits in rats to which transgenic technology cannot be easily applied. 